\title{
Evaluación del efecto cicatrizante de una crema y pomada a base de aguamiel sobre la espalda de ratas Wistar
}

\author{
Evaluation of the healing effect of a cream and ointment based on aguamiel on the back of Wistar rats
}

\author{
A. E. González-Martínez iDa M. G. Ortiz-José iDa, I. J. Rodríguez-Meléndez iDa, Y. de las M. Gómez y Gómez iDb \\ A. J. Hurtado-Mariles iDa,b M. I. Jiménez-Zúñiga (iDa,b,*
}

${ }^{a}$ Universidad Tecnológica de Tecámac, División Químico Biológicas, 55749, Tecámac, Estado de México, México.

${ }^{\mathrm{b}}$ Instituto Politécnico Nacional, Unidad Profesional Interdisciplinaria de Biotecnología, Departamento de Bioprocesos, Laboratorio de Farmacología, 07340, Ciudad de México, México.

\begin{abstract}
Resumen
El aguamiel o agua de agave es una bebida que se extrae del interior de diferentes especies de género Agave como lo es el agave pulquero (Agave salmiana Haw), maguey de montaña (A. atrovirens Karw) y el agave americano (A. americana L.). Estas son plantas originarias de México, presentes en zonas semiáridas y templadas del altiplano. El aguamiel contiene saponinas, un metabolito secundario el cual se caracteriza por su estructura, ya que contiene una aglicona esteroidal o terpenoide y una o más cadenas de polisacáridos. El presente proyecto evaluó el efecto cicatrizante del aguamiel (Agave spp.) en una crema y pomada al 5\% y $10 \%$ de concentración, las pruebas in vivo se realizaron sobre la espalda de ratas Wistar. La pomada y crema se colocaron sobre incisiones de $1.5 \mathrm{~cm}$ de largo con una profundidad de $0.2 \mathrm{~cm}$ en la parte externa de la medula espinal. Los tratamientos se aplicaron dos veces al día, con crema y pomada al $5 \%$ y $10 \%$, sulfadiazina de plata y el grupo control. Los resultados obtenidos muestran que el proceso de cicatrización con crema y pomada ocurrió en menor tiempo en comparación con el grupo de sulfadiazina de plata, ya que el tiempo de cicatrización del primer tratamiento fue de siete días, a diferencia del segundo tratamiento que fue de quince días.
\end{abstract}

Palabras Clave:

Agua miel, cicatrización, saponinas.

\begin{abstract}
The aguamiel or water is a drink that is extracted from the interior of different species of the genus Agave, such as the "pulquero agave" (Agave salmiana Haw), mountain maguey (A. Atrovirens Karw) and the American agave (A. americana L.). These are native plants from Mexico, which are present in semi-arid and temperate zones of the highlands. Aguamiel contains saponins, a secondary metabolite which is characterized by its structure, since it contains a steroidal or terpenoid aglycone and one or more polysaccharide chains. The healing effect of aguamiel (Agave spp.) was evaluated in the present project, using a cream and an ointment at 5\% and $10 \%$ concentration. In vivo tests were carried out on the back of Wistar rats. The ointment and cream were placed over $1.5 \mathrm{~cm}$ long incisions with a depth of $0.2 \mathrm{~cm}$ in the external part of the spinal cord. The treatments were applied twice a day with cream and ointment at $5 \%$ and $10 \%$, silver sulfadiazine and the control group. The results obtained show that the healing process with cream an ointment occurred in less time compared to the silver sulfadiazine group, since the healing time of the first treatment was seven days, unlike the second treatment, which was fifteen days.
\end{abstract}

Keywords:

Aguamiel, healing, saponins.

\section{Introducción}

México es considerado centro de origen del Agave y el principal centro agrícola de América, resultando en su diversificación comercial bajo esquemas de selección y manejo humano, se generó primero por la selección natural, y desde hace unos 10,000 años, por la selección humana, y actualmente los pobladores del país y el mundo son quienes aprovechan de los beneficios. El Agave salmiana es una especie endémica con gran importancia biológica, cultural y económica, la cual cuenta con

\footnotetext{
*Autor para la correspondencia: mjimenez.zuniga@ hotmail.com

Correo electrónico: evelynglezx3@gmail.com (Areli Evelyn González-Martínez), maira.joseo@gmail.com (Maira Guadalupe Ortiz-Jose), israelrodmel@gmail.com (Israel Jesús Rodríguez-Meléndez), ygomez@ipn.mx (Yolanda de las Mercedes Gómez y Gómez), alejandroj_hm@hotmail.com (Alejandro Jonathan Hurtado Mariles),
} mjimenez.zuniga@ hotmail.com (Marcos Ignacio Jiménez-Zuñiga). 
características que la distinguen de los otros agaves (GarcíaMarín, 2007).

El Agave spp, se ha utilizado en México desde la época precolombina como una fuente de alimento y fuente de bebida. En la actualidad, el Agave tiene una relevancia económica en las industrias del tequila, mezcal y pulque. Del Agave spp se obtiene el aguamiel que es la savia dulce y comestible obtenida de las pencas maduras y específicas, como el Agave salmiana. En México se consume el aguamiel para mejorar ciertas condiciones como la diabetes, ya que se ha reportado que mejora el control de la glucosa en el cuerpo de igual forma se ha evaluado los efectos biológicos que esta tiene en el cuerpo humano (Leal-Díaz et al., 2016).

El aguamiel es el principal componente para la producción del pulque, por lo que el principal estado con mayor producción es Hidalgo, seguido de Tlaxcala y el Estado de México, ya que en el año de 2017 el estado de Hidalgo aporto el $69.6 \%$ del pulque a nivel nacional que equivale a 217.7 millones de litros (Crespo, 2015; Servicio de información agroalimentaria y pesquera, 2018).

El aguamiel o agua de agave es una bebida que se extrae del interior de diferentes especies de género Agave como lo es el agave pulquero (Agave salmiana Haw), maguey de montaña ( $A$. atrovirens Karw.) y el agave americano (A. americana L.). El aguamiel contiene saponinas, que son un metabolito secundario el cual se caracteriza por su estructura, se encuentran presentes en plantas medicinales que se categorizan según las cadenas de azúcar en su estructura y son llamadas con los sufijos "mono",dis-", y "tris-"; las saponinas monodesmásidas contienen una sola cadena de azúcar unida al C-3, las bidesmosídicas tienen dos cadenas de azúcar generalmente conectadas con un éter en el C-3 y las saponinas trismesodícas las cuales presentan tres cadenas de azúcares unidos con un enlace éster a un C-28 o un enlace éter en un C-26 (García-Marín, 2007), contienen una aglicona esteroidal o terpenoide y una o más cadenas de polisacáridos (Leal-Díaz et al., 2016). Las saponinas se presentan en la etapa de maduración del agave y depende de su especie, ya que varía el tipo de saponinas presentes en ellos, por ejemplo, las esteroideas, de las cuales las más destacables son la hecogenina y la gentrogenina y en el aguamiel la más común es la kammogenina, dentro de las propiedades del aguamiel están las actividades fungistáticas, antimicóticas y antimicrobianas (Güçlü-Üstündağ et al., 2007; Crespo, 2015).

La cicatrización es un método de reparación de heridas mediado por proteínas solubles (citosinas y factores de crecimiento) y células encargadas de la proliferación celular para el establecimiento de tejido lesionado. Hay dos tipos de herida, la primera consiste en un método mecánico para cerrar la herida (suturas, cintas, etc.), la cual ocurre dentro de las 12 a 24 horas al haber cerrado la de la piel, esto por causa del trauma severo que se haya presentado en la zona o quemadura y cuyo tiempo depende de la extensión de la herida y la segunda se caracteriza por no regenerar la arquitectura normal de cicatrización, (Valencia, 2010).

\section{Metodología}

El aguamiel se recolectó en el poblado de San Juan Teotihuacan de Arista en el Estado de México. Se recolectaron $20 \mathrm{~mL}$ de aguamiel por planta en tugo Falcon de 3 diferentes plantas de agave. Se etiquetaron con la fecha y el lugar de recolección.
Posteriormente se guardaron (durante 5 horas, para evitar la fermentación) en refrigeración a $8{ }^{\circ} \mathrm{C}$ para su posterior uso.

Posteriormente se analizó la presencia de saponinas con el método cualitativo del tamiz fitoquímico, donde se realizó la prueba de espuma y la reacción de Liebermann-Burchard al aguamiel para comprobar la presencia de saponinas (Valencia et al., 2010).

Para la prueba de altura y estabilidad de espuma se colocó 1 $\mathrm{mL}$ del aguamiel y se agitó vigorosamente. Se tomó la altura de la espuma, si es que presenta espuma (es positivo si la espuma alcanza una altura de $8-10 \mathrm{~mm}$ y es estable por $30 \mathrm{~min}$ ). Posteriormente se realizó la reacción de Lieberman Bouchard, se concentró $0.5 \mathrm{~mL}$ de aguamiel hasta $0.2 \mathrm{~mL}$, después se agregó 2 gotas de anhidro acético y se esterificó con 2 gotas de ácido sulfúrico concentrado (el color azul o verde en la interfase indica la presencia de saponinas esteroideas, si la coloración es rosa, rojo o magenta o violeta presencia de saponinas triterpenoides).

Para la formulación de la crema y pomada a base de aguamiel, se preparó una formulación de $25 \mathrm{~g}$ para cada una.

Para la formulación de la crema se agregó $16 \mathrm{~g}$ de aceite de almendras, $5.5 \mathrm{~g}$ de lanolina, $0.5 \mathrm{~g}$ de almendra de cacao, $1.5 \mathrm{~g}$ de cera de abeja y $26.5 \mathrm{~mL}$ de agua caliente. En un vaso de precipitados calentado a baño María , se vierte la cera de abeja y la de cacao, agitando constantemente hasta que se fundan perfectamente. Sin dejar de agitar añade la lanolina y el aceite de almendras, se deja calentar por $10 \mathrm{~min}$, y una vez retirado se añade el agua tibia y se mezcla contantemente hasta la mezcla se enfríe y tome una apariencia cremosa (aproximadamente $20 \mathrm{~min}$ ), posteriormente se añadió el 5 y $10 \%$ de aguamiel $(132.5 \mathrm{~mL}$ y $66.25 \mathrm{~mL})$.

Para la formulación de la pomada se añadieron $2.5 \mathrm{~g}$ de cera blanca y $16.25 \mathrm{~g}$ de lanolina, se difundió la cera en baño María, posteriormente se difundió la lanolina lentamente con ayuda de un agitador, una vez difundidas la cera y lanolina se mezclaron ambas mezclas hasta realizar una mezcla homogénea, por último se agregó el aguamiel y se siguió agitando hasta el proceso de envasado, cuando la mezcla se encuentra totalmente homogénea y comienza el proceso de solidificación.

Se determinó el pH y la prueba de homogeneidad a la crema y pomada de acuerdo con la Farmacopea de los Estados Unidos Mexicanos (2011).

Los ensayos in vivo para el proceso de cicatrización se realizaron utilizando 12 ratas Wistar macho de entre 250-300 g de peso corporal. Se anestesiaron con pentobarbital sódico y posteriormente se depilaron en el área dorsal (espalda), donde se realizaron 2 heridas asépticas de $1.5 \mathrm{~cm}$ de diámetro con un bisturí estéril, los tratamientos colocados fueron Crema y pomada al 5 y $10 \%$ de aguamiel, sulfadiazina de plata y control sin tratamiento. La aplicación de los tratamientos se realizó 2 veces al día durante 7 días, posteriormente se monitoreo el proceso de cicatrización a diario (González-Escobar 2002). El uso y cuidado de los animales se llevó a cabo siguiendo los lineamientos establecidos en la NOM-062-ZOO-1999 que establece las especificaciones técnicas para la producción, cuidado y uso de los animales de laboratorio.

\section{Resultados y discusión}

Del tamiz fitoquímico se obtuvo positivo en la prueba de estabilidad de espuma, ya que la espuma tuvo una altura de 8.5 
$\mathrm{mm}$ al igual que la reacción de Liebermann-Burchard, donde se observó la presencia de saponinas esteroidales, de acuerdo con las pruebas cualitativas. La estructura de las saponinas consiste en una aglicona no polar llama sapogenina, unida a uno o más restos de polisacáridos, que pueden unirse como una, dos o tres cadenas laterales. Estas saponinas pueden tener una gran diversidad estructural debido a la variabilidad en la aglicona, a la composición del polisacárido y a su ubicación. Las agliconas contribuyen a los sistemas de defensa de la planta para protegerla ante cualquier agente extraño, se sabe que contiene propiedades antimicrobianas y antifúngicas. $\mathrm{Y}$ algunas de estas pueden tener beneficios a la salud humana. Además, se ha estudiado el efecto antimicrobiano en células (Leal -Díaz et al., 2015).

Por otro lado, se ha informado que los extractos de las hojas y los residuos de Agave sisalana tienen actividad antifúngica. Otras especies como Agave lechuguilla, Agave picta, Agave scabra y Agave lophanta se ha demostrado que presentaron efectos antimicrobianos contra bacterias patógenas. Levaduras y algunos hongos (Medina-Galván et al., 2018).

Santos-Zea et al., (2012) ha reportado compuestos derivados de glicósidos esteroideos como tigogenina, smilagenina, gitogenina, clorogenina, hecogenina, sarsapogenina, neotigogenina y hongguanggenina, cuya estructura contiene una molécula de azúcar que se sabe desempeña un papel importante dentro de las actividades antimicrobianas.

En las pruebas fisicoquímicas el pH obtenido en la crema fue de 5.5 realizadas con el potenciómetro, mientras que la pomada tuvo un $\mathrm{pH}$ de 6.5 a 7, de acuerdo con la literatura las pomadas y las cremas deben tener un $\mathrm{pH}$ ligeramente ácido a neutro (Ibáñez et al., 1970).

Para los resultados de homogeneidad se evaluó la apariencia, observación visual, olor y uso sobre la piel. La crema presentó una apariencia cremosa y con un buen color (claro debido al aguamiel), la pomada presentó una apariencia sólida y de color amarillento, la observación visual fue agradable, ya que tiene un color y una buena apariencia. De acuerdo con Mosquera et al., (2012) los atributos de evaluación sobre la piel son:

- Suavidad: piel suave y agradable al tacto.

- Firmeza de la piel: mostró una estabilidad en el mantenimiento de sus características que permiten mantener la resistencia y estructura.

(Ibáñez et al., 1970)

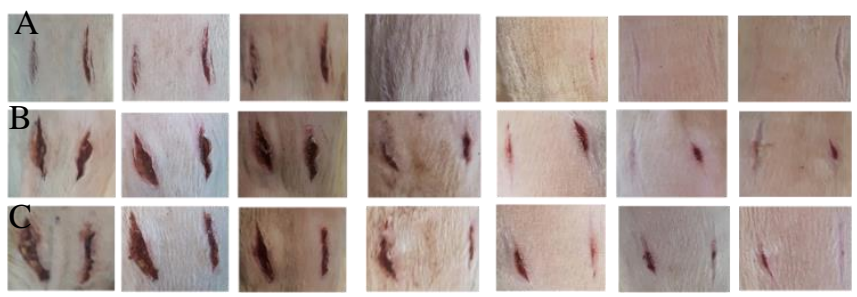

Figura 1: Resultados de la prueba de cicatrización en la espalda de ratas Wistar.

En la figura 1 se observan los tratamientos aplicados, se realizaron dos heridas una izquierda y una derecha con diferentes tratamientos, en la letra A se observa de lado izquierdo la pomada al $5 \%$ y al derecho la crema al $5 \%$. En la letra B de lado izquierdo se encuentra la pomada al $10 \%$ y de lado derecho el fármaco (sulfadiazina de plata). En la letra $\mathrm{C}$ de lado izquierdo control sin tratamiento y derecho crema al $10 \%$.

Los resultados de las pruebas in vivo demostraron que los tratamientos aplicados a base de aguamiel comenzaron más rápido el proceso de cicatrización en las heridas realizadas sobre la espalda de las ratas, se puede observar que el tratamiento con la pomada y crema al $5 \%$ el proceso es más rápido que el fármaco que se utilizó como control. El fenómeno de la cicatrización transcurre en diversas etapas: hemostasia, inflamación, proliferación, remodelación y maduración de la cicatriz. De acuerdo con estudios después de 3 o 4 días ocurre la proliferación de fibroblastos que comienzan a migrar hacia la matriz provisional del coágulo (Martínez et al., 2016).

De acuerdo con Valencia (2010), el proceso de cicatrización es complejo y depende de la interacción de los elementos celulares que se producen como las proteínas necesarias para la reacción inflamatoria y la reparación del tejido. Existen dos tipos de cicatrización, los de primera intención, que ocurre en un periodo dentro de las primeras 12 a 24 horas después de que ha sido cerrada la herida y el segundo, de segunda intención, el cual tiene una característica de no alcanzar a regenerar la arquitectura normal con la que cuenta la piel debido a la pérdida de tejido. De acuerdo con los resultados obtenidos el proceso de cicatrización se podría deber a los de primera intención, proceso que podría seguir la crema y pomada a base de aguamiel, debido a que el proceso de cicatrización comenzó entre el segundo y tercer día después de realizar la herida.

\section{Conclusiones}

El proceso de cicatrización ocurrió en menor tiempo para los tratamientos de contenían aguamiel en la formulación de la pomada y la crema en comparación con el fármaco utilizado como control.

Las saponinas presentes en el aguamiel pudieran tener un efecto cicatrizante debido a que contiene saponinas, a las cuales se les atribuye un efecto detergente y cicatrizante.

\section{Agradecimientos}

Este trabajo ha sido realizado parcialmente gracias al apoyo del Laboratorio de Farmacología del Departamento de Bioprocesos de la Unidad Profesional Interdisciplinaria de Biotecnología del Instituto Politécnico Nacional.

\section{Referencias}

Comisión Permanente de la Farmacopea de los Estados Unidos Mexicanos. (2011). Farmacopea de los Estados Unidos Mexicanos. Secretaría de Salud.

Crespo, G., (2015). Maguey para fines medicinales. Periodismo crítico. México.

García-Marín, P. C., (2007). E5 En lo ancestral hay futuro: Del tequila, los mezcales y otros agaves. CICY. México.

González Escobar, R., (2002). Modelos experimentales para la evaluación de la acción cicatrizante de medicamentos. Revista Cubana de Farmacia 36, 189-196.

Güçlü-Üstündağ, Ö. Mazza, G., (2007). Saponins: properties, applications and processing. Critical reviews in food science and nutrition 47, 231 258.DOI: 10.1080/10408390600698197 
Ibáñez, S. Suñé, J. M., (1970). Estudio experimental del pH en sistemas dispersos de interes farmaceutico: iii estudio del pH en pomada de f.e.ix, especialidades facrmaceuticas $y$ de elaboracion propia. Ars pharmaceutica (internet) 11,30.

Leal-Díaz, A. M; Santos-Zea, L; Martínez-Escobedo, H. C; Guajardo-Flores, D; Gutiérrez-Uribe, J. A; Serna-Saldivar, S. O., (2015). Effect of Agave americana and Agave salmiana ripeness on saponin content from aguamiel (agave sap). Journal of agricultural and food chemistry 63,39243930. DOI: 10.1021/acs.jafc.5b00883

Leal-Díaz, A. M; Noriega, L. G; Torre-Villalvazo, I; Torres, N; AlemánEscondrillas, G; López-Romero, P; Avila-Nava, A., (2016). Aguamiel concentrate from Agave salmiana and its extracted saponins attenuated obesity and hepatic steatosis and increased Akkermansia muciniphila in C57BL6 mice. Scientific reports 6, 34242. DOI: 10.1038/srep34242

Martínez, J. L. M., Pérez, V. M., Castillo, O. P., Gutierrez, M. C., Bernal, O. J. L., Cabrera, M. G. C., Ruíz, D. H., (2016). Implante de catgut para cicatrización de heridas inducidas (Modelo de cicatrización en la rata). REDVET. Revista Electrónica de Veterinaria 17, 1-10.

Medina-Galván, M.I; Bernardino-Nicanor, A; Castro-Rosas, J; Negrete, M. D. L. L. X; Rodríguez, E. C. B; González-Cruz, L., (2018). Antimicrobial and antioxidant activity of flower scape extracts of Agave salmiana: effect of the extraction solvent and development stage. Research Journal of Biotechnology 13,1-9.
Mosquera, T., Noriega, P., Tapia, W., Pérez, S. H., (2012). Evaluación de la eficacia cosmética de cremas elaboradas con aceites extraídos de especies vegetales Amazónicas: Mauritia Flexuosa (Morete), Plukenetia Volubilis (Sacha Inchi) y Oenocarpus Bataua (Ungurahua). La Granja 16, 14-22. DOI: $10.17163 / \operatorname{lgr} . n 16$

Norma Oficial Mexicana NOM-062-ZOO-1999, Especificaciones técnicas para la producción, cuidado y uso de los animales de laboratorio. Secretaría de Agricultura, Ganadería, Desarrollo Rural, Pesca y Alimentación. Mexico.

Santos-Zea, L; Leal-Diaz, A; Cortés-Ceballos, E; Gutierrez-Uribe J., (2012). Agave (Agave spp.) and its traditional products as a source of bioactive compounds. Current Bioactive Compounds 8, 218-231.DOI: $10.2174 / 157340712802762410$

Servicio de información agroalimentaria y pesquera., (2018). Maguey pulquero: el estado de Hidalgo destacó en 2017 con $69.6 \%$ de la producción nacional. Obtenido de https://www.gob.mx/siap/articulos/maguey-pulquero.

Unidad Profesional Interdisciplinaria de Biotecnología. Manual de Prácticas de Laboratorio Tecnología Farmacéutica II (1er). México: Instituto Politécnico Nacional.

Valencia, B. C., (2010). Cicatrización: proceso de reparación tisular. Aproximaciones terapéuticas. Revista Investigaciones Andina 12, 85 - 98. DOI:10.33132/01248146.242

Valencia, G., Garín, M., (1a Ed.), (2010). Manual de prácticas de productos naturales. México D.F. 